\title{
Erratum to: Distributed dislocation approach for cracks in couple-stress elasticity: shear modes
}

\author{
P. A. Gourgiotis - H. G. Georgiadis
}

Published online: 22 July 2011

(C) Springer Science+Business Media B.V. 2011

\section{Erratum to: Int J Fract (2007) 147:83-102 DOI 10.1007/s10704-007-9139-5}

The authors wish to apologize for any inconvenience caused due to the fact that erroneous versions of Eqs. (14), (58), and (60a,b) were published. Please find on this page the correct Eqs. (14), (58), and (60a,b) that should be regarded by the reader as the final versions

$$
\begin{aligned}
\omega_{i j}= & \frac{1}{2}\left(\partial_{i} u_{j}-\partial_{j} u_{i}\right) \\
\tau_{x z}= & -\frac{\mu b}{2 \pi r} \sin \theta-\frac{\mu b(1+\beta)}{8 \pi \ell}(\sin 3 \theta+\sin \theta) \\
& \times K_{1}(r / \ell)+\frac{\mu b(1+\beta)}{2 \pi r} \\
& \times\left[\frac{2 \ell^{2}}{r^{2}}-K_{2}(r / \ell)\right] \sin 3 \theta \\
\tau_{y z}= & \frac{\mu b}{2 \pi r} \cos \theta+\frac{\mu b(1+\beta)}{8 \pi \ell}(\cos 3 \theta-\cos \theta) \\
& \times K_{1}(r / \ell)-\frac{\mu b(1+\beta)}{2 \pi r} \\
& \times\left[\frac{2 \ell^{2}}{r^{2}}-K_{2}(r / \ell)\right] \cos 3 \theta,
\end{aligned}
$$

The online version of the original article can be found under doi:10.1007/s10704-007-9139-5.

P. A. Gourgiotis · H. G. Georgiadis ( $\square)$

Mechanics Division, National Technical University of Athens, Zographou Campus, Zographou 15773, Greece e-mail: georgiad@ central.ntua.gr

$$
\begin{aligned}
m_{y y}= & -m_{x x}=\frac{\mu \ell^{2}(1+\beta) b}{\pi r^{2}} \cos 2 \theta \\
& -\frac{3 \mu b \ell^{2}(1+\beta)^{2}}{\pi r^{2}}\left(\frac{2 \ell^{2}}{r^{2}}-K_{2}(r / \ell)\right) \cos 4 \theta \\
& +\frac{\mu b(1+\beta)^{2}}{2 \pi} K_{2}(r / \ell) \cos 4 \theta \\
& -\frac{\mu b(1+\beta)^{2}}{8 \pi} K_{0}(r / \ell)(3 \cos 4 \theta+1)
\end{aligned}
$$

where all symbols are defined in the paper. The above misprints do not affect the results of the paper. 